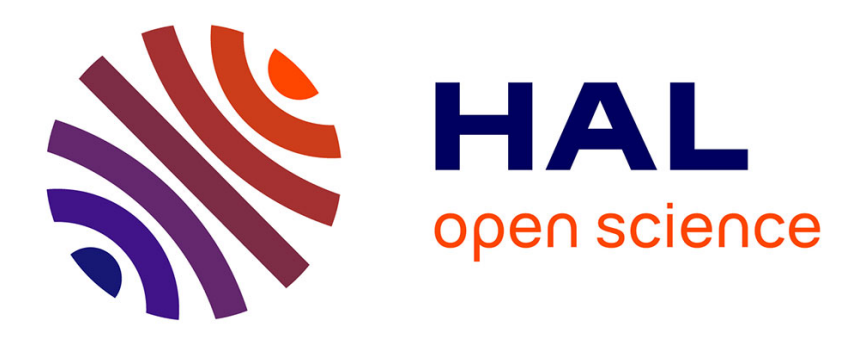

\title{
Multivariable model predictive control of a catalytic reverse flow reactor
}

\author{
Pascal Dufour, Youssoufi Touré
}

\section{To cite this version:}

Pascal Dufour, Youssoufi Touré. Multivariable model predictive control of a catalytic reverse flow reactor. Computers \& Chemical Engineering, 2004, 28 (11), pp.2259-2270. 10.1016/j.compchemeng.2004.04.006 . hal-00351742v2

\section{HAL Id: hal-00351742 \\ https://hal.science/hal-00351742v2}

Submitted on 21 Jan 2009

HAL is a multi-disciplinary open access archive for the deposit and dissemination of scientific research documents, whether they are published or not. The documents may come from teaching and research institutions in France or abroad, or from public or private research centers.
L'archive ouverte pluridisciplinaire HAL, est destinée au dépôt et à la diffusion de documents scientifiques de niveau recherche, publiés ou non, émanant des établissements d'enseignement et de recherche français ou étrangers, des laboratoires publics ou privés. 
This document must be cited according to its final version which is published in a journal as:

P. Dufour ${ }^{1}$, Y. Touré2,

"Multivariable model predictive control of a catalytic reverse flow reactor", Computers and Chemical Engineering, ISSN: 0098-1354

28(11), pp. 2259-2270, 2004.

http://dx.doi.org/10.1016/j.compchemeng.2004.04.006

All open archive documents of Pascal Dufour are available at: http://hal.archives-ouvertes.fr/DUFOUR-PASCAL-C-3926-2008

The professional web page (Fr/En) of Pascal Dufour is:

http://www.lagep.univ-lyon1.fr/signatures/dufour.pascal

1

Université de Lyon, Lyon, F-69003, France; Université Lyon 1;

CNRS UMR 5007 LAGEP (Laboratoire d'Automatique et de GEnie des Procédés),

43 bd du 11 novembre, 69100 Villeurbanne, France

Tel +33 (0) 472431845 - Fax +33 (0) 472431699

http://www-lagep.univ-lyon1.fr/ http://www.univ-lyon1.fr http://www.cnrs.fr

2

Université d'Orléans,

UPRES EA 2078 LVR (Laboratoire de Vision et de Robotique ),

63 Av de Lattre de Tassigny, 18020 Bourges Cedex, France

http://www.bourges.univ-orleans.fr/rech/lvr/ 


\title{
Multivariable Model Predictive Control of a
}

\section{Catalytic Reverse Flow Reactor}

\author{
Dufour P. ${ }^{\mathrm{a}, 1}$, Touré Y. ${ }^{\mathrm{b}}$ \\ ${ }^{\mathrm{a}}$ LAGEP, Université Claude Bernard Lyon 1, UMR CNRS 5007 \\ 43, Bd du 11 Novembre 1918, 69622 Villeurbanne Cedex, France. \\ ${ }^{\mathrm{b}}$ LVR, Université d'Orléans, UPRES EA 2078 \\ 63, Av de Lattre de Tassigny, 18020 Bourges Cedex, France.
}

\begin{abstract}
This paper is devoted to the multiple input multiple output (MIMO) model predictive control (MPC) of a catalytic reverse flow reactor (RFR). The RFR aims to reduce, by catalytic reaction, the amount of volatile organic compounds (VOCs) released in the atmosphere. The peculiarity of this process is that the gas flow inside the reactor is periodically reversed in order to trap the heat released during the reaction. The objective of this paper is to propose a solution to avoid the limitation seen in a previous study for the single input single output (SISO) control case. It was dealing with the impossibility to avoid degradation of the catalytic elements
\end{abstract}


due to the excessive heating induced by the exothermic reaction. In order to overcome this issue, a ratio of the inlet (cold) gas flow is now bypassed into the central zone. This allows introducing a second manipulated variable: the dilution rate. The phenomenological model considered here for the MIMO MPC of the RFR is obtained from a rigorous first principles modeling. The resulting accurate nonlinear partial differential equation (PDE) model can be a drawback for the model based controller implementation. To overcome this issue, we use a MPC strategy previously developed: it combines a two phase approximation of the PDE model in an internal model control (IMC) structure. This strategy allows using a less accurate model and a less time-consuming control algorithm. Finally, efficiency of the control approach is shown in simulation.

Key words:

Catalytic reverse flow reactor, nonlinear distributed parameter system, model predictive control, internal model control.

\section{Introduction}

Problems of environment pollution due to the industrial production are receiving increased attention: due to public regulations, VOCs discharge in the atmosphere is strictly limited. Even if the definition of VOCs is blurred, it includes noxious products which chemical reactivity is likely to influence atmospheric pollution. For this reason, they are the source of a lot of environ-

$\overline{1}$ Corresponding author: email dufour@lagep.univ-lyon1.fr, phone (33) 4724318 78, fax (33) 472431699 
mental problems including: acid rains, woods wasting, greenhouse effect and health troubles. Therefore, the VOCs emission reduction represents a priority, especially since the problem is connected with a large field of activities from large-scale factories to small and medium-sized firms like dry cleaners.

An experimental process was build-up in the LGPC ${ }^{2}$. It is a RFR that allows high temperatures in catalyst bed whereas the inlet and outlet gas stream temperatures are close to ambient temperature. This process is useful for experimental validation of solution for issues like: physical phenomena that influence purification efficiency, optimal size of each elements, process behavior in industrial use and control.

In a previous work, the SISO control case was treated [9]. The aim was to control the process such that the outlet gas concentration released in the atmosphere was maintained below a maximum level fixed by public regulations. This control strategy was based on a PDE model, an IMC structure and a MPC framework. According to various regimes, it was shown that the proposed controller was able to tune correctly the control action, i.e. the heating power at the core of the reactor. In the meantime, for a particular regime, the temperature inside the reactor was exceeding a threshold temperature that reflects the deterioration of the catalytic elements. Therefore, the aim of this paper is to provide a MIMO control framework to solve this problem. This requires modifying the PDE model and the control problem to account for the second manipulated variable: the cooling action.

The paper is structured has follows: in section 2, the RFR, the new PDE model and the new MIMO problem are presented. Section 3 aims to remind

$\overline{2}$ Laboratoire de Génie des Procédés Catalytiques, UMR CNRS 2214, CPE Lyon, France. 
the control strategy previously developed for the SISO control case [9]. In section 4, simulation results for the MIMO control strategy of the RFR are discussed.

\section{RFR: modeling and control problem}

\subsection{Destruction of VOCs with the RFR}

The aim of the RFR is to reduce, by catalytic reaction, the amount of VOCs released in the atmosphere. The peculiarity of this process is that the gas circulation sense is periodically reversed (Fig. 1). It allows high temperatures in catalyst bed whereas the inlet and outlet gas stream temperatures are close to ambient temperature. The operation procedure of this RFR [20] is described below:

- A semi-cycle begins as follows: the gas flows through the first thermal monolith. It is made up of cordierite and of $n b_{c}$ canals where the gas flows. The shape of its section is a nest. The increase of gas temperature in the canals is due to the heat exchange with the cordierite. No reaction takes place and cooling action is possible;

- The gas then passes through the first catalytic monolith. This one is like the first thermal monolith but with catalytic elements (platinum and other noble metals) layers on the canals surface. With these elements, the exothermic chemical reaction takes place, inducing increase of temperature in the cordierite and a concentration of pollutant drop. A cooling action is possible;

- The gas flows now in the empty central zone where two control actions are 
both provided: heating and cooling sources;

- The reaction continues in a second catalytic monolith and finally the gas reaches a second thermal monolith where no reaction occurs but where the heat of reaction is exchanged from the gas to the cordierite.

- At the end of this semi-cycle, the switch of the four servo valves reverses the flow rate inside the monoliths. A second semi-cycle, identical to the first one, starts but in the reverse circulation sense;

- Since the circulation sense has changed, the polluted gas passes first through the previous second thermal monolith. In this zone, the gas temperature increases using the heat previously accumulated in the monolith during the first semi-cycle: This is the saving mode of the process;

- At the end of this second semi-cycle, the flow rate in the reactor is again reversed thanks to the servo valves switch and a new complete cycle begins.

According to the operating conditions, various problematic behaviors can take place:

- Insufficiently polluted gas at the RFR inlet causes, by its low heat release during the reaction, the extinction of the reactor;

- With a strongly polluted gas at the RFR inlet, the release of heat due to the reaction can deteriorate the catalysts and produces thermal overheating.

to overcome extinction and overheating, several technical solutions have been proposed [25] and are used in this work:

- Extinction: fuel addition in the gas or energy addition in the central zone.

- overheating: use of a bypass to redirect some amount of the gas or injection of cold gas in the central zone. 


\subsection{Modeling}

VOCs combustion in a RFR has been studied in packed bed or monolith by various authors (see [20] for a general review). Models are based on standard heat and mass balances and most often deal with an adiabatic RFR at stationary periodic state $[25,16,34]$. They assume the analogy with a counter current reactor for the RFR at high frequency, which allows estimating simple RFR characteristics. In [8], authors study the effects of an external electrical heater supply for very lean mixture. The dynamic aspects of the RFR have been less examined. A simple linear dynamic model accounting for heat losses and dilution has been developed in [30]. Budman et al. [4] have developed a nonlinear dynamic model.

Quiet similar to Budman et al.'s model, in our approach, one assumes that the adiabatic RFR has dynamic behavior, that gas velocity is constant and that there is no pressure loss. For modeling, heat and mass balances are described along the independent space variable $z$ following the flow sense. Due to high thermal capacity and short residence time in the reactor, the phenomena in the canals are assumed instantaneous. In the solid parts, it is also assumed that the dynamic of the concentration of pollutant is negligible with respect to the dynamic of the temperature. The following physical quantities are considered in each of the five zones (Fig. 2) of the reverse flow reactor (two thermal monoliths, two catalytic monoliths and the central part):

- the concentration of pollutant $C_{g}(z, t)$ and the temperature $T_{g}(z, t)$ of the gas inside the canals;

- the concentration of pollutant $C_{s}(z, t)$ and the temperature $T_{s}(z, t)$ of the cordierite along the solid parts. 
Limitation of the SISO control strategy was dealing with the impossibility to avoid that the temperature inside the reactor exceeds a maximum limit [9]. It deals with limitation for the use of the reactor due to the degradation of the catalytic elements at high temperature. In order to overcome this issue, a ratio of the inlet (cold) gas flow is now bypassed into the central zone. This allows introducing a new manipulated variable: the dilution rate $\alpha$ defined by

$$
\alpha=1-\frac{u_{g . b y p a s s e d}}{u_{g}}
$$

where $u_{g}$ is the inlet gas velocity and $u_{\text {g.bypassed }}$ is the velocity of the inlet gas directly bypassed into the central zone. The dilution rate $\alpha$ therefore ranges between 0 (maximum cooling and minimum amount of gas cleaned up) and 1 (no cooling and maximum amount of gas treated). Therefore, the control problem is now stated here as a MIMO control problem. From the modeling point of view, the evolutions of the previous model [9] are therefore concerned with a change of gas flow rate expression for $z \in\left[0, z_{Z C}[\right.$ and with a new term in the central zone. This leads to the following model:

Remark 2.1 To account for the periodic sense inversion, the model is written in four steps over a complete cycle $\left.T=] \tau, \tau+T_{\text {cycle }}\right]$ where $\tau=n T_{\text {cycle, }},(n$ is an integer).

Description and value of the model parameters may be found in [9].

$1 /$ during the first semi-cycle $(t \in] \tau, \tau+\frac{T_{\text {cycle }}}{2}[)$ :

- at the inlet $(z=0)$ : 


$$
\left\{\begin{array}{l}
T_{g}=T_{g \cdot \text { inlet }}(t) \\
C_{g}=C_{g \cdot \text { inlet }}(t) \\
\frac{\partial T_{s}}{\partial z}=0 \\
C_{s}=0
\end{array}\right.
$$

- in the first thermal zone (for $z \in \Omega_{M T 1}$ ), where the cooling manipulated variable (MV) $\alpha$ is accounted for:

$$
\left\{\begin{array}{l}
\frac{\partial T_{g}}{\partial z}=\frac{h a_{c}}{\alpha u_{g} \rho_{g} c_{p_{g}}}\left(T_{s}-T_{g}\right) \\
C_{g}=C_{g}(0, t) \\
\frac{\partial T_{s}}{\partial t}=\frac{\lambda}{\rho_{s t} c_{p_{s}}} \frac{\partial^{2} T_{s}}{\partial z^{2}}+\frac{h a_{c}}{(1-\varepsilon) \rho_{s t} c_{p_{s}}}\left(T_{g}-T_{s}\right) \\
C_{s}=0
\end{array}\right.
$$

- at the boundary between the first thermal zone and the first catalytic zone $\left(z=z_{M T 1}\right):$

$$
\left\{\begin{array}{l}
T_{g \mid z^{-}}=T_{g \mid z^{+}} \\
C_{g}=C_{g}(0, t) \\
\left.\frac{\partial T_{s}}{\partial z}\right|_{z^{-}}=\left.\frac{\partial T_{s}}{\partial z}\right|_{z^{+}} \\
C_{s}=0
\end{array}\right.
$$

- in the first catalytic zone $\left(z \in \Omega_{M C 1}\right)$, where the cooling $\mathrm{MV} \alpha$ is 
accounted for:

$$
\left\{\begin{array}{l}
\frac{\partial T_{g}}{\partial z}=\frac{h a_{c}}{\alpha u_{g} \rho_{g} c_{p_{g}}}\left(T_{s}-T_{g}\right) \\
\frac{\partial C_{g}}{\partial z}=\frac{k_{d} a_{c}}{\alpha u_{g}}\left(C_{s}-C_{g}\right) \\
\frac{\partial T_{s}}{\partial t}=\frac{\lambda}{\rho_{s c} c_{p_{s}}} \frac{\partial^{2} T_{s}}{\partial z^{2}}+\frac{h a_{c}}{(1-\varepsilon) \rho_{s t} c_{p_{s}}}\left(T_{g}-T_{s}\right)+\frac{\left(-\Delta H_{r}\right) k^{\infty} a_{c}}{(1-\varepsilon) \rho_{s c} c_{p_{s}}} e^{\frac{-E a}{R T_{s}}} C_{s} \\
C_{s}=\frac{1}{1+\frac{k^{\infty}}{k_{d}} e^{\frac{-E a}{R T_{s}}}} C_{g}
\end{array}\right.
$$

- at the outlet of the first catalytic zone $\left(z=z_{M C 1}\right)$, where the cooling MV $\alpha$ is accounted for:

$$
\left\{\begin{array}{l}
\frac{\partial T_{g}}{\partial z}=\frac{h a_{c}}{\alpha u_{g} \rho_{g} c_{p_{g}}}\left(T_{s}-T_{g}\right) \\
\frac{\partial C_{g}}{\partial z}=\frac{k_{d} a_{c}}{\alpha u_{g}}\left(C_{s}-C_{g}\right) \\
\frac{\partial T_{s}}{\partial z}=0 \\
C_{s}=\frac{1}{1+\frac{k^{\infty}}{k_{d}} e \frac{-E a}{R T_{s}}} C_{g}
\end{array}\right.
$$

- in the central zone (for $z \in \Omega_{Z C}$ ), the heating MV $P_{\text {res }}$ and the cooling MV $\alpha$ are accounted for:

$$
\left\{\begin{array}{l}
\frac{\partial T_{g}}{\partial z}=\frac{1}{S \alpha u_{g} \rho_{g} c_{p_{g}} L_{Z C}} P_{r e s}-\frac{1-\alpha}{\alpha} T_{a i r} \\
C_{g}=C_{g}\left(z_{M C 1}, t\right)
\end{array}\right.
$$

- at the central zone outlet $\left(z=z_{Z C}\right)$ : 


$$
\left\{\begin{array}{l}
\frac{\partial T_{g}}{\partial z}=\frac{h a_{c}}{u_{g} \rho_{g} c_{p g}}\left(T_{s}-T_{g}\right) \\
\frac{\partial C_{g}}{\partial z}=\frac{k_{d} a_{c}}{u_{g}}\left(C_{s}-C_{g}\right) \\
\frac{\partial T_{s}}{\partial z}=0 \\
C_{s}=\frac{1}{1+\frac{k^{\infty}}{k_{d}} e^{\frac{-E a}{R T_{s}}}} C_{g}
\end{array}\right.
$$

- in the second catalytic zone (for $z \in \Omega_{M C 2}$ ):

$$
\left\{\begin{array}{l}
\frac{\partial T_{g}}{\partial z}=\frac{h a_{c}}{u_{g} \rho_{g} c_{p_{g}}}\left(T_{s}-T_{g}\right) \\
\frac{\partial C_{g}}{\partial z}=\frac{k_{d} a_{c}}{u_{g}}\left(C_{s}-C_{g}\right) \\
\frac{\partial T_{s}}{\partial t}=\frac{\lambda}{\rho_{s c} c_{p_{s}}} \frac{\partial^{2} T_{s}}{\partial z^{2}}+\frac{h a_{c}}{(1-\varepsilon) \rho_{s t} c_{p_{s}}}\left(T_{g}-T_{s}\right)+\frac{\left(-\Delta H_{r}\right) k^{\infty} a_{c}}{(1-\varepsilon) \rho_{s c} c_{p_{s}}} e^{\frac{-E_{a}}{R T_{s}} C_{s}} \\
C_{s}=\frac{1}{1+\frac{k^{\infty}}{k_{d}} e^{\frac{-E a}{R T_{s}}}} C_{g}
\end{array}\right.
$$

- at the boundary between the second catalytic zone and the second thermal zone $\left(z=z_{M C 2}\right)$ :

$$
\left\{\begin{array}{l}
T_{g \mid z^{-}}=T_{g \mid z^{+}} \\
C_{g}=C_{g}\left(z_{M C 2^{-}}, t\right) \\
\left.\frac{\partial T_{s}}{\partial z}\right|_{z^{-}}=\left.\frac{\partial T_{s}}{\partial z}\right|_{z^{+}} \\
C_{s}=\frac{1}{1+\frac{k^{\infty}}{k_{d}} e^{\frac{-E_{a}}{R T_{s}}}} C_{g}
\end{array}\right.
$$

- in the second thermal zone (for $z \in \Omega_{M T 2}$ ): 


$$
\left\{\begin{array}{l}
\frac{\partial T_{g}}{\partial z}=\frac{h a_{c}}{u_{g} \rho_{g} c_{p_{g}}}\left(T_{s}-T_{g}\right) \\
C_{g}=C_{g}\left(z_{M C 2}, t\right) \\
\frac{\partial T_{s}}{\partial t}=\frac{\lambda}{\rho_{s t} c_{p_{s}}} \frac{\partial^{2} T_{s}}{\partial z^{2}}+\frac{h a_{c}}{(1-\varepsilon) \rho_{s t} c_{p_{s}}}\left(T_{g}-T_{s}\right) \\
C_{s}=0
\end{array}\right.
$$

- at the process outlet $\left(z=z_{M T 2}\right)$ :

$$
\left\{\begin{array}{l}
\frac{\partial T_{g}}{\partial z}=\frac{h a_{c}}{u_{g} \rho_{g} c_{p_{g}}}\left(T_{s}-T_{g}\right) \\
C_{g}=C_{g}\left(z_{M C 2}, t\right) \\
\frac{\partial T_{s}}{\partial z}=0 \\
C_{s}=0
\end{array}\right.
$$

2/ At the end of the semi-cycle $\left(t=\tau+\frac{T_{c y c l e}}{2}\right)$, the change of the circulation sense leads to a state permutation ( $L_{t o t}$ is the total length of the reactor):

$$
\left\{\begin{array}{l}
T_{g}\left(z, \tau+\frac{T_{\text {cycle }}}{2}\right)=T_{g}\left(L_{t o t}-z, \tau+{\frac{T_{\text {cycle }}}{2}}^{-}\right) \\
C_{g}\left(z, \tau+\frac{T_{\text {cycle }}}{2}\right)=C_{g}\left(L_{t o t}-z, \tau+{\frac{T_{c y c l e}}{2}}^{-}\right) \\
T_{s}\left(z, \tau+\frac{T_{c y c l e}}{2}\right)=T_{s}\left(L_{t o t}-z, \tau+{\frac{T_{c y c l e}}{2}}^{-}\right) \\
C_{s}\left(z, \tau+\frac{T_{c y c l e}}{2}\right)=C_{s}\left(L_{t o t}-z, \tau+{\frac{T_{c y c l e}}{2}}^{-}\right)
\end{array}\right.
$$

$3 /$ During the second semi-cycle $(t \in] \tau+\frac{T_{c y c l e}}{2}, \tau+T_{\text {cycle }}[)$, equations (2) to (12) are again valid. 
4/ At the end of the cycle $\left(t=\tau+T_{\text {cycle }}\right)$, another state permutation takes place and a new complete cycle begins:

$$
\left\{\begin{array}{l}
T_{g}\left(z, \tau+T_{\text {cycle }}\right)=T_{g}\left(L_{t o t}-z, \tau+T_{\text {cycle }}^{-}\right) \\
C_{g}\left(z, \tau+T_{\text {cycle }}\right)=C_{g}\left(L_{t o t}-z, \tau+T_{\text {cycle }}^{-}\right) \\
T_{s}\left(z, \tau+T_{\text {cycle }}\right)=T_{s}\left(L_{t o t}-z, \tau+T_{\text {cycle }}^{-}\right) \\
C_{s}\left(z, \tau+T_{\text {cycle }}\right)=C_{s}\left(L_{t o t}-z, \tau+T_{\text {cycle }}^{-}\right)
\end{array}\right.
$$

The model clearly exhibits a strong nonlinear behavior due to the catalytic reaction, due to the MVs $P_{\text {res }}$ and $\alpha$ and due to the periodic discontinuity. For more details about the modeling, reader is referred to [30].

Therefore, we consider the class of MIMO one dimensional nonlinear parabolic PDE system $\left(S_{N L}\right)$ with scalar control:

$$
\left\{\begin{array}{l}
\frac{\partial \underline{x}(z, t)}{\partial t}=F_{d}(\underline{x}(z, t), \underline{u}(t), t) \forall z \in \Omega, t>0 \\
F_{b}(\underline{x}(z, t), \underline{u}(t), t)=0 \forall z \in \partial \Omega, t>0 \\
\underline{x}(z, 0)=\underline{x}_{i} \forall z \in \Omega \cup \partial \Omega \\
\underline{y}_{m}(t)=C(t) \underline{x}(z, t) \forall z \in \partial \Omega, t>0
\end{array}\right.
$$

where $z$ is the independent space variable, $\Omega$ is the spatial domain and $\partial \Omega$ its boundary, $t$ is the independent time variable. For all $t \geq 0, \underline{x}(z, t)$ is the state vector in a Hilbert Space, $\underline{u}(t)$ is the control or MV vector in $\mathbb{R}^{2}, \underline{y}_{m}(t)$ is the model output vector in $\mathbb{R}^{p} . F_{d}$ and $F_{b}$ are nonlinear operator and $C$ is a linear operator. 
Assumption 1 There exists $\underline{u}(t)=\underline{u}_{0}(t)$ leading to the particular representation $\left(S_{0}\right)$ of $\left(S_{N L}\right)$ described by the triplet $\left\{\underline{u}(t)=\underline{u}_{0}(t), \underline{x}(z, t)=\underline{x}_{0}(z, t), \underline{y}_{m}(t)=\right.$ $\left.\underline{y}_{0}(t)\right\}$, for all $t \geq 0$.

Variations around $\left(S_{0}\right)$ are given by:

$$
\left\{\begin{array}{l}
\underline{u}(t)=\underline{u}_{0}(t)+\Delta \underline{u}(t) \\
\underline{x}(t)=\underline{x}_{0}(t)+\Delta \underline{x}(t) \\
\underline{y}_{m}(t)=\underline{y}_{0}(t)+\Delta \underline{y}_{m}(t)
\end{array}\right.
$$

where sufficiently small variations about $\left(S_{0}\right)$ are described by the time-varying linearized PDE model $\left(S_{T V L}\right)$ :

$$
\left\{\begin{array}{l}
\frac{\partial \underline{\underline{x}}(z, t)}{\partial t}=A_{d}^{x}(t) \Delta \underline{x}(z, t)+A_{d}^{u}(t) \Delta \underline{u}(t) \forall z \in \Omega, t>0 \\
A_{b}^{x}(t) \Delta \underline{x}(z, t)+A_{b}^{u}(t) \Delta \underline{u}(t)=0 \forall z \in \partial \Omega, t>0 \\
\Delta \underline{x}(z, 0)=\underline{0} \forall z \in \Omega \cup \partial \Omega \\
\Delta \underline{y}_{m}(t)=C(t) \Delta \underline{x}(z, t) \forall z \in \Omega \cup \partial \Omega, t>0
\end{array}\right.
$$

where the time-varying linear operators $A_{d}^{x}(t), A_{d}^{u}(t), A_{b}^{x}(t)$ and $A_{b}^{u}(t)$ are obtained from the linearization of $\left(S_{N L}\right)$ about the behavior described by $\left(S_{0}\right)$ $[14]$. 


\subsection{MIM0 Control Problem}

The control problem for the RFR tackled in this paper relates first to the statutory maximum amount of VOC that can be released into the atmosphere at the process outlet. This is stated as an output constraint: one has to ensure that the concentration of pollutant at the process outlet is lower than a maximum level fixed by public regulations:

$$
C_{g}\left(z_{M T 2}, t\right) \leq C_{g \cdot \max } \forall t>0
$$

From a practical point of view, it is replaced by a constraint on the mean output concentration of pollutant calculated over a past time window $T$ :

$$
\left\{\begin{array}{l}
C_{g . \text { outlet }}(t)=\frac{1}{T} \int_{t-T}^{t} C_{g}\left(z_{M T 2}, \tau\right) d \tau \\
C_{g . \text { outlet }}(t) \leq C_{g \cdot \max }
\end{array}\right.
$$

Secondly, in order to avoid deterioration of the catalytic elements, temperature inside the process has to be maintained under a maximum value, which was not possible in the previous SISO control study [9]. On the other side, most of the reaction takes place at the inlet of the first catalytic monolith and is instantaneous $[24,25,30]$. The second output constraint is therefore:

$$
T_{s}\left(z_{M T 1}, t\right) \leq T_{s \cdot \max } \forall t>0
$$

Moreover, manipulated variables (electrical power $P_{\text {res }}$ and dilution rate $\alpha$ ) have to be tuned such that:

- Over consumption of electrical power fed into the central zone is minimized,

- A maximum amount of gas is cleaned up. 


\section{Controller design}

The RFR control problem presented here deals with the control of a nonlinear parabolic PDE system characterized by complex nonlinearities and periodic discontinuities in the spatial domain and at the boundary as well. Even with only one spatial dimension, control of PDE systems are not often treated, especially in the nonlinear case. Explicitly, transport reaction phenomena with significant diffusive and convective phenomena are typically characterized by severe nonlinearities and spatial variations, and are naturally described by partial differential equations. Examples of such processes include tubular reactors, packed bed reactors, absorption columns, drying or curing processes. The large number of real applications that results in such PDE models constitutes another motivation for this work. In control theory, due to the complexity of the problem, relatively few studies are devoted to the control of processes explicitly characterized by a PDE model. Even if various methods are proposed to control such distributed parameter systems, there is no general framework yet. In order to implement, with a computer, a low order model based controller, the original PDE model is usually simplified into an ordinary differential equation (ODE) model. Such a finite dimension approximation is based on finite differences, finite volume method, orthogonal collocation or Galerkin's method. Other works utilized properties of the initial PDE system before finite dimension controller synthesis: Recently, Christofides developed order reduction by partitioning the eigen spectrum of the operator of the PDE system [6,12] and methods based on approximate inertial manifold for spatial discretization of the PDE $[5,2]$. Other works for controller synthesis of nonlinear PDE systems are based on symmetry groups, infinitesimal generators and invariant con- 
ditions $[26,15]$. Concerning $[17,35]$, finite dimensional controller are obtained through model reduction based on various methods: singular value decomposition, Karhunen-Loéve expansion or eigenfunction method. With this method, a framework is provided with proof of closed-loop stability for the QDMC of a PDE system [36]. In [3], stability conditions for closed loop control of linear PDE with finite dimension controller are given in time domain and frequency domain through semigroup analysis. In [33], based on semigroup theory, proofs were given for the closed loop stability of PI control for a linear PDE system. Moreover, the control strategy used here has to be able to be implementable for on-line control and has to account for natural physical limitations or specifications (constraints) inherent to many processes. An advanced model based control strategy, developed for ordinary differential equation systems, is well dedicated to solve this constrained problem: model predictive control $[1,23,21,31,29]$. MPC, or receding horizon control, refers to a class of control algorithms in which a dynamic process model is used to predict and optimize process performance. Today, MPC has become an advanced control strategy widely used in industry. Indeed, MPC is well suited for high performance control since constraints can be explicitly incorporated into the formulation of the control problem. MPC was applied for a few PDE systems where accurate high order dimension models are accounted for $[10,27,32,18]$. Very recently [36], singular value decomposition and Karhunen-Loéve expansion was used in a QDMC framework.

Few papers are devoted to RFR control: [24] deals with this problem with ignition and extinction phenomena, whereas [7] gives some guidelines for the control of such process accounting for auto thermal and overheating phenomena. Recently, to avoid extinction and overheating, [19] propose a simple switching control law strategy by on-line tuning of the switching time. 
Unfortunately, temperature may exceed, in large transient peaks, the maximum temperature allowed and overall results are not optimized. Until now, the first complete control study has been written by Budman et al. [4] where a first-principles pseudo-homogeneous one-dimensional model is provided. In this paper, a parametric study of the reactor allows characterizing the working mode of the reactor with respect to two manipulated variables: the coolant flow rate and the cycle time. Moreover, Budman et al. developed two SISO control approaches where coolant flow rate is the MV when the temperature and the concentration at the reactor inlet were assumed constant. First, a PID controller, based on a local linear model is tuned, is given. Even if the use of PID controller is quiet simple, the main drawback is that there is no guarantee of constraints satisfaction. Secondly, a feed forward controller is given but it is not usable during transient conditions and it is not robust with respect to modeling errors. Concerning our previous work [9], we presented SISO control results where the aim was to minimize the consumption of electrical power accounting for the constraint dealing with limitation of pollutant released at the process outlet. Limitation of this strategy was concerned with the impossibility to control overheating, which is addressed here. Compared with Budman et al.'s work [4], in our approach, the cycle time can not be chosen as a manipulated variable: indeed, the parametric study [30] showed that the residence time of the gas inside the reactor has to be small (a few seconds) in order to trap the heat inside the reactor. Cycle time is therefore constant and tuned to $20 \mathrm{~s}$. Moreover, simulation results cover here more realistic cases since gas concentration of pollutant at the reactor inlet (i.e the main input disturbance) is time-varying and unmeasured. This is not the case in Budman et al.'s work. 


\subsection{PDE model based IMC-MPC strategy}

In this paper, we use a method [9] that aims to reduce, during the sampling period, the on-line calculation time due to the optimization task resolution involved by the PDE model based MPC strategy. Indeed, from a practical point of view, one of the drawbacks of MPC is the computational time aspect, especially when the model becomes more complex and more accurate, which is the case with the RFR model presented here. Indeed, the model is intended to predict the future dynamic behavior of the process output over a finite prediction horizon and has to be solved during the on-line constrained optimization problem resolution.

The method used to reduce this computational time [9] is reminded here. The idea is to use on-line two models: the nonlinear parabolic PDE model $\left(S_{0}\right)$ solved off-line combined with a time-varying linearized PDE model $\left(S_{T V L}\right)$ solved on-line during the optimization task. The use of the IMC structure (Fig. 3) allows using less accurate (hence less time consuming) finite dimension approximation of the time-varying linearized PDE model $\left(S_{T V L}\right)$ since it corrects errors due to the open loop model predictions. Indeed, for the model approximation, two tuning strategies are possible for the number of nodes used in the finite difference method:

- First possibility, the number of nodes is tuned to a "small" value ("small" with respect to the large number of nodes to find an accurate approximation of the nonlinear parabolic PDE system solution). In this case, less time is required for the model resolution and more time can be spent for the optimization task. But since the MPC formulation is an open loop predictive optimization procedure, large errors due to the PDE model solution ap- 
proximation used in the optimization task may degrade closed loop control results.

- The second possibility for the tuning is to increase the number of nodes to find a more accurate approximated solution of the PDE system. This leads to increase the time needed to solve the model and to decrease the time dedicated to the optimization task resolution. The drawback is that the optimizer may not have the time to find a solution during the sampling time.

Clearly speaking, a trade-off exists for this tuning: even if it leads to a loss of accuracy in open loop input output behavior, it allows reaching the closed loop performances specified while providing a less time-consuming resolution task. This will be shown in simulation results.

A transformation method is used to handle hard input constraints. It allows translating explicit constraints based on the optimization argument $\underline{u}$ (and only on the optimization argument) as new equations for new unconstrained argument $\underline{p}[28]$. Here, we propose to enlarge this method to cover magnitude constraints and velocity constraints as well (acceleration rate constraints may also be accounted for) [9]: it guarantees that the actions always respect their constraints (magnitude, velocity). In order to account for output constraints as soft constraints (for which violation may be allowed [29]), we adopt the exterior penalty method [13] used in nonlinear programming where a positive defined weighted penalty term is added to the initial cost function involved in the MPC problem. The penalty method transforms the problem into an unconstrained problem by substituting a penalty function for each constraint. Therefore, the constrained solution might be approached from the unfeasible side of the constraints: this relative drawback can be an advantage in the 
case where, sometimes, no solution for the constrained problem exists and constraint relaxation has therefore to be allowed. According to the stochastic variations of the inlet gas concentration, such unfeasibility issue may happen during the control of the RFR.

\section{Simulation results}

Finally, the control problem is formulated in the MPC framework as:

$$
\left\{\begin{array}{l}
\min _{P_{\text {res }}(k), \alpha(k)} J\left(P_{\text {res }}(k), \alpha(k)\right)=\left(\left[\frac{P_{\text {res }}(k)-P_{\text {res.min }}}{P_{\text {res.max }}-P_{\text {res.min }}}\right]^{2}+\left[\frac{\alpha(k)-\alpha_{\max }}{\alpha_{\text {max }}-\alpha_{\min }}\right]^{2}\right) \\
\text { with constraints on magnitude and velocity of the MVs: } \\
P_{\text {res.min }} \leq P_{\text {res }}(j) \leq P_{\text {res.max }} \quad \forall j \in J_{0}^{N_{p}-1}=\left\{k, k+N_{p}-1\right\} \\
\Delta P_{\text {res.min }} \leq P_{\text {res }}(j)-P_{\text {res }}(j-1) \leq \Delta P_{\text {res.max }} \quad \forall j \in J_{0}^{N_{p}-1} \\
\alpha_{\text {min }}<\alpha(j) \leq \alpha_{\text {max }} \quad \forall j \in J_{0}^{N_{p}-1} \\
\Delta \alpha_{\text {min }} \leq \alpha(j)-\alpha(j-1) \leq \Delta \alpha_{\text {max }} \quad \forall j \in J_{0}^{N_{p}-1} \\
\text { with constraints on controlled variables: } \\
C_{g}\left(z_{M T 2}, j\right) \leq C_{g \cdot \max } \quad\left(\text { with } j \in\left\{k+1, k+N_{p . c}\right\}\right) \\
T_{s}\left(z_{M T 1}, j\right) \leq T_{s . \max } \quad\left(\text { with } j \in\left\{k+1, k+N_{p . t}\right\}\right)
\end{array}\right.
$$

where $k$ is the actual discrete time index, $j$ is the discrete time index. $N_{p . c}$ and $N_{p . t}$ are respectively the prediction horizon for the output constraint stated on the concentration of pollutant and the prediction horizon for the output constraint stated on the temperature in the solid, $N_{p}=\max \left(N_{p . c}, N_{p . t}\right)$. In 
order to decrease the computational burden, control horizon tuning is one. Even it is true that is restricts the degree of freedom to solve the optimization problem, it allows decreasing the computational time, which is an important issue here.

\subsection{Simulation conditions}

The runs are realized in the following conditions:

(1) For both the nonlinear system $\left(S_{0}\right)$ solved off-line and the time-varying linearized PDE model $\left(S_{T V L}\right)$ solved on-line, a finite volume approach is used to approximate the solution. Since the number of volumes chosen directly influences the accuracy of the solution and the computational time, it is also chosen as a tuning parameter. One want to underline here that a compromise can be found between a relatively inaccurate finite dimension approximation of the time-varying linearized PDE system $\left(S_{T V L}\right)$ (but that can be solved faster) and a relatively good approximation of the time-varying linearized PDE system $\left(S_{T V L}\right)$ (that needs more computation time for its resolution) in order to get efficient closed loop control results.

(2) After trial error tests, the model linearization is done around the nonlinear behavior $\left(S_{0}\right)$ obtained with, $\forall t \geq 0$ :

$$
\left\{\begin{array}{l}
P_{\text {res.0 }}(t)=520 \mathrm{~W} \\
\alpha_{0}(t)=0.95 \\
C_{g . \text { inlet. } 0}(t)=2 \times 10^{-3} \text { mol. } \mathrm{m}^{-3}
\end{array}\right.
$$


(3) Concerning the 3 input disturbances, the inlet flow rate and the inlet gas temperature are assumed constant and measured, $\forall t \geq 0$ :

$$
\left\{\begin{array}{l}
Q_{g}(t)=100 \mathrm{~m}^{3} \cdot \mathrm{h}^{-1} \\
T_{g \text {.inlet }}(t)=20^{\circ} \mathrm{C}
\end{array}\right.
$$

whereas the inlet gas concentration of pollutant is stochastic (only its mean value is known) in three zones, $\forall t \geq 0$ :

$$
\left\{\begin{array}{l}
\text { Low level: } 1 \times 10^{-3} \text { mol. } m^{-3}<C_{g . \text { inlet }}(t)<3 \times 10^{-3} \text { mol. } m^{-3} \\
\text { Medium level: } 4 \times 10^{-3} \text { mol. } m^{-3}<C_{g . \text { inlet }}(t)<6 \times 10^{-3} \text { mol.m }{ }^{(234)} \\
\text { High level: } 16 \times 10^{-3} \text { mol. } m^{-3}<C_{g . \text { inlet }}(t)<18 \times 10^{-3} \text { mol.m } m^{-3}
\end{array}\right.
$$

The inlet gas concentration is acting as an input disturbance with a strong influence over closed-loop performances. Therefore, robustness property of the controller has to be important and is shown here.

(4) Constraints bounds are:

$$
\left\{\begin{array}{l}
P_{\text {res.max }}=3000 \mathrm{~W}, P_{\text {res.min }}=0 \mathrm{~W} \\
\alpha_{\max }=1, \alpha_{\min }=0 \\
\Delta P_{\text {res.max }}=+1500 \mathrm{~W} . \mathrm{s}^{-1}, \Delta \alpha_{\max }=+1 \mathrm{~s}^{-1} \\
\Delta P_{\text {res.min }}=-1500 \mathrm{~W} . \mathrm{s}^{-1}, \Delta \alpha_{\min }=-1 \mathrm{~s}^{-1} \\
C_{g \max }=4.7 \times 10^{-4} \mathrm{~mol} . \mathrm{m}^{-3}, T_{\text {s.max }}=673^{\circ} \mathrm{C}
\end{array}\right.
$$

(5) For the output constraints given in Eq. (19), the length of the time window $T$ is $20 \mathrm{~min}$. 
(6) The sample rate $T_{e}$ value is half of the cycle period (round trip) $T_{\text {cycle }}$ value:

$$
\left\{\begin{array}{l}
T_{\text {cycle }}=20 \mathrm{~s} \\
T_{e}=0.5 T_{\text {cycle }}
\end{array}\right.
$$

(7) Simulations are run using a $500 \mathrm{MHz}$ CPU, a FORTRAN code and the ddaspg subroutine of IMSL library for the time integration (Petzold-Gear BDF method).

(8) Concerning the tuning of the horizons, even if this is important in any MPC implementation, this influence is not underline here. Indeed, during the simulations results detailed below, one want to underline the role of the finite dimension approximation of the solution of the time-varying linearized PDE system $\left(S_{T V L}\right)$ involved in the on-line MPC formulation. Therefore, prediction horizons have be tuned such that the process dynamics that directly influence the optimization results are sufficiently accounted for.

Remark 4.1 To see a smooth trend of the electrical power fed into the central zone, the mean value, calculated since the beginning of each run (including the warm-up period) at $t=0 s$, is depicted instead of the value found at each time.

Remark 4.2 For the evolution of the concentration of pollutant at the process outlet depicted, the discontinuity that occurs at 1200s is due to the initial memorization needed to evaluate system (19). 


\subsection{Open loop control results}

Concerning the resolution of nonlinear PDE system $\left(S_{N L}\right)$ used to simulate the process, it is obtained with a finite volume approach using 803 volumes. The open loop process behavior obtained is depicted Fig. 4 (snapshots of the temperature profile in the solid). The profile at $t=0 \mathrm{~s}$ is the profile obtained at the end of a preheating period, where no polluted gas is fed into the reactor: The aim of this period is to allow the catalytic elements to warm-up such that they are sufficiently warm to be able to react with the polluted gas. According to the snapshots, one can first see the distributed aspect of the process: Between the inlet $(z=0)$ and the outlet of the first catalytic monolith $\left(z=z_{M C 2}\right)$, i.e. over a length of $45 \mathrm{~cm}$, the temperature increases by $500{ }^{\circ} \mathrm{C}$ (Fig. 4). Moreover, one can see the interest of the cooling since the temperature decreases in the center of the reactor.

Concerning computational aspect for this open loop control simulation, one has first to remind that, for this process, the sampling time $T_{e}$ is $10 \mathrm{~s}$. It is therefore small with respect to the time needed to solve the finite dimensional approximation of the nonlinear PDE system $\left(S_{N L}\right)$ : indeed, with a $500 \mathrm{MHz}$ PC, 18 seconds of the open loop process evolution, in average, might be simulated during 1 second used by the CPU. That clearly penalizes the direct use of the nonlinear PDE system $\left(S_{N L}\right)$ during a predictive control framework, since prediction horizons have to be tuned sufficiently large to cover the dynamic evolution of the process and that some iterations are required to find a solution to the penalized optimization problem.

Given these open loop control results, for this process, this gives motivation for the use of the proposed time-varying linearized PDE model based MPC 
strategy.

\subsection{Closed loop control results}

The use of the reactor in 3 different levels of inlet gas concentration (detailed in (24) ) allows, with simulation, to see 3 various use of the reactor. The inlet gas concentration is strongly stochastic and has as clearly a very strong impact over closed loop performances since it acts as an input disturbance. Following runs will also show how the proposed controller is robust with respect to this disturbance. It also allows to see the influence of the finite dimension approximation of the time-varying linearized PDE system $\left(S_{T V L}\right)$ solved during the on-line optimization task.

- Run \#1 deals with the low level of pollution at the RFR inlet. In order to achieve the cleaning up objective described by the output constraint (19) depicted Fig. 5, an external source of energy is necessary and electrical power is fed into the reactor (Fig. 6): about $12 \%$ of the full capacity of electrical supply tends asymptotically to be reached. Due to the stochastic variations of the inlet gas concentration and its evident large influence over the control performance, the constraints is noisy but is fulfilled and not satured. In the case of constant and known input disturbances, runs show that this constraint is asymptotically satured from the constrained side. Due to the low content of pollutant and hence the low thermal energy released during the reaction, there is no overheating issue. Therefore, the dilution rate is always equal to its maximum value: the maximum amount of gas is treated. According to the results depicted Fig. 5, one can see that output constraint is fulfilled, whatever the model approximation is. But, the drawback of using 
a small number of volumes (67) is that it leads to an over consumption of electrical power (Fig. 6). On the contrary, from a correct minimum value (243), increasing the number of volumes does not change global closed loop performance: the same solution is found for the electrical power (Fig. 6) and for the constrained output as well (Fig. 5) even if approximation errors exist (e.g.: instantaneous concentration of pollutant at the outlet depicted Fig. 7). Moreover, the control algorithm can be implemented for on-line control since the resolution of the optimization procedure is always less than the sample time (this is also the case for the next runs).

- Run \#2 deals with the medium level of pollution at the RFR inlet. About $1 \%$ of the full capacity of electrical supply tends asymptotically to be reached (Fig. 8). Indeed, the amount of pollutant becomes sufficiently high such that the heat generated during the reaction (and trapped inside the reactor) allows reaching a particular regime: the autothermic regime where no external source of energy is needed to satisfy the output constraint (19) (Fig. 9). The dilution rate is maximal since there is no overheating issue. Concerning the influence of the number of volumes, it is again clearly underlined. Fig. 8 shows that a small number of volumes (67) leads to a large over consumption of energy. This is avoided, increasing the number of volumes to 243: up to 5 times less electrical power is required, whereas the output constraint (19) is still fulfilled and is closer to the threshold prescribed than with 67 volumes. Increasing the number of volumes does not change again global closed loop performance.

- Run \#3 deals with the high level of pollution at the RFR inlet. As expected, the autothermic regime is reached: no external source of energy is needed and the output constraint (19) is always checked: almost no pollutant is present at the process outlet. In the meantime, due to the large amount 
of pollutant at the process inlet, thermal energy trapped inside the reactor produces a large increase of temperature in the monoliths (Fig. 10). Overheating of the reactor is avoided by the tuning of the dilution rate (Fig. 11). This penalizes the gas velocity inside the reactor, since it is asymptotically decreased to $90 \%$ of its maximum value: hence less gas is treated. One can see the influence of the number of volumes: if it is important, the optimizer has not enough time to find a solution that fulfilled the constraint (20) (see Fig. 10 and 11 with 403 volumes). By decreasing the number of volumes, a solution can be computed during the sampling time and the constraint bound is always reached, even if the constraint might not be strictly fulfilled. This is due to the stochastic behavior of the inlet gas concentration, that has a large impact over control results and that is not accounted for in the control algorithm. In the case of constant input disturbance, runs show that this constraint bound is asymptotically reached without any overshoot.

\section{Conclusion}

This paper was dealing with the MIMO MPC of a catalytic reverse flow reactor. This process is used to decrease noxious VOC amount in gas released in the atmosphere. The complexity of this process included distributed aspect, nonlinear dynamic behavior and periodic reversing of the circulation of gas. The MPC was based on a two-phase approximation of the first-principle nonlinear parabolic PDE model in an IMC framework. Runs showed that the use of the proposed controller leads to interesting closed loop performances, even with a large input disturbance. Regarding implementation issue for such controller, even if a relatively large-scale model needs to be solved in the MPC strategy 
with a small sample time of $10 \mathrm{~s}$, the on-line implementation was shown to be possible. Two advances are currently under study: first, the development of the control framework based on a new countercurrent pseudo-homogeneous model [11] which is faster to compute. Secondly, in order to improve the closedloop performances, the use of the on-line estimation of the stochastic inlet gas concentration acting as a strong input disturbance [11]. 


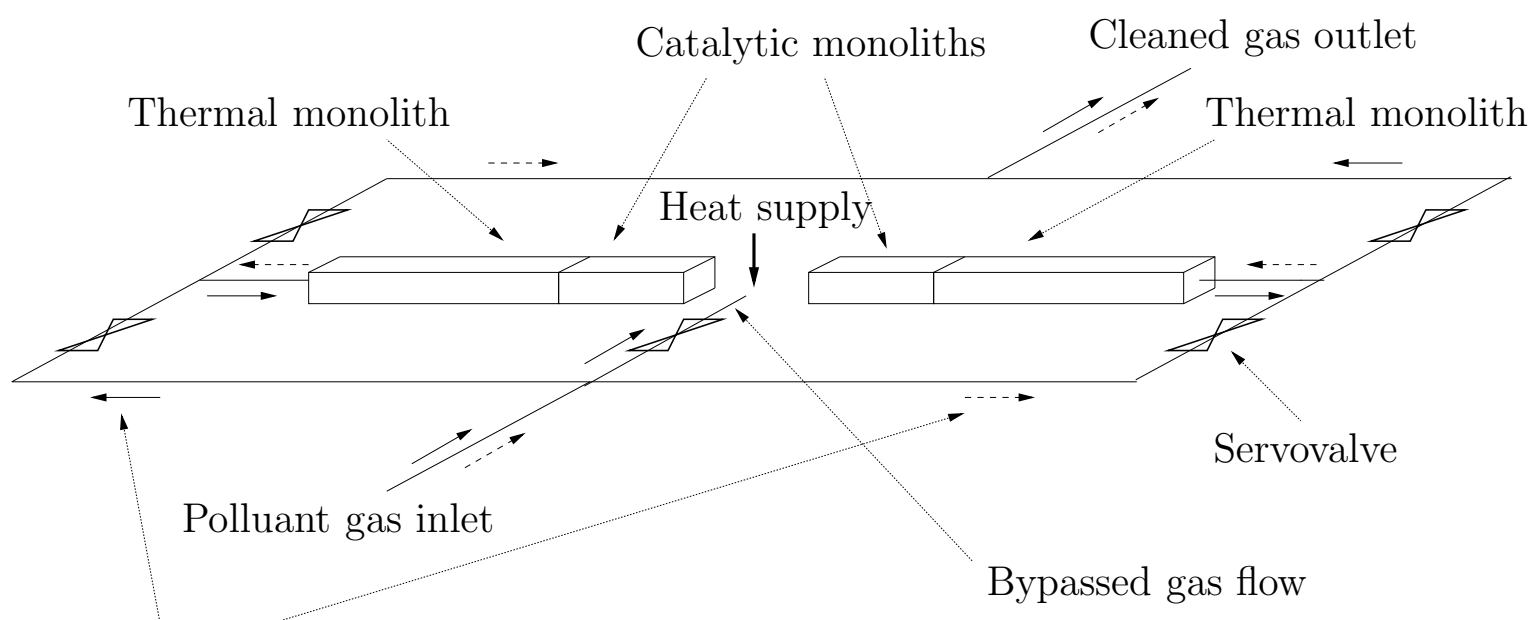

Possible flowing out senses

Fig. 1. Principle scheme for the catalytic reverse flow reactor.

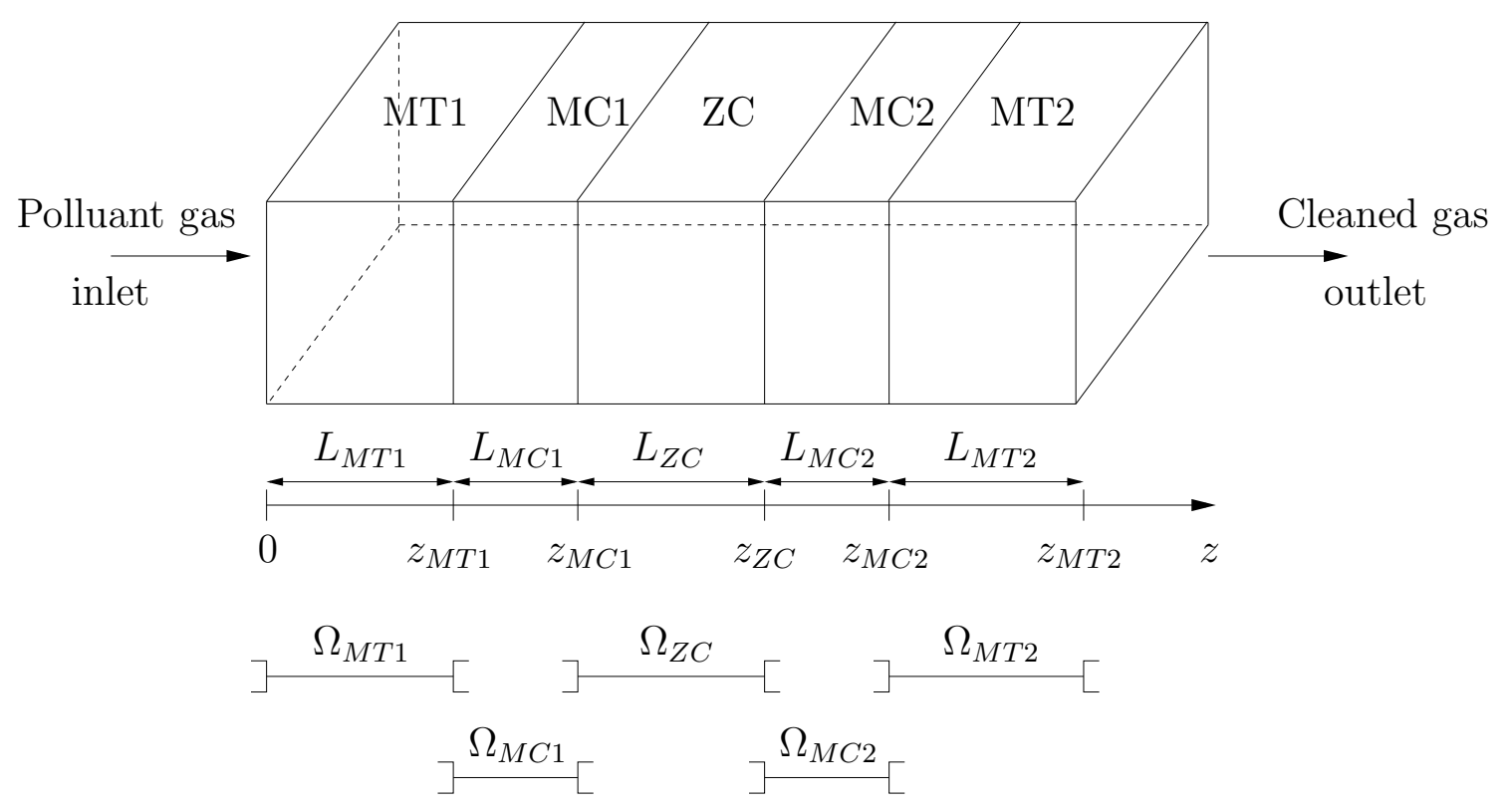

Fig. 2. Spatial discretization in the reactor. 


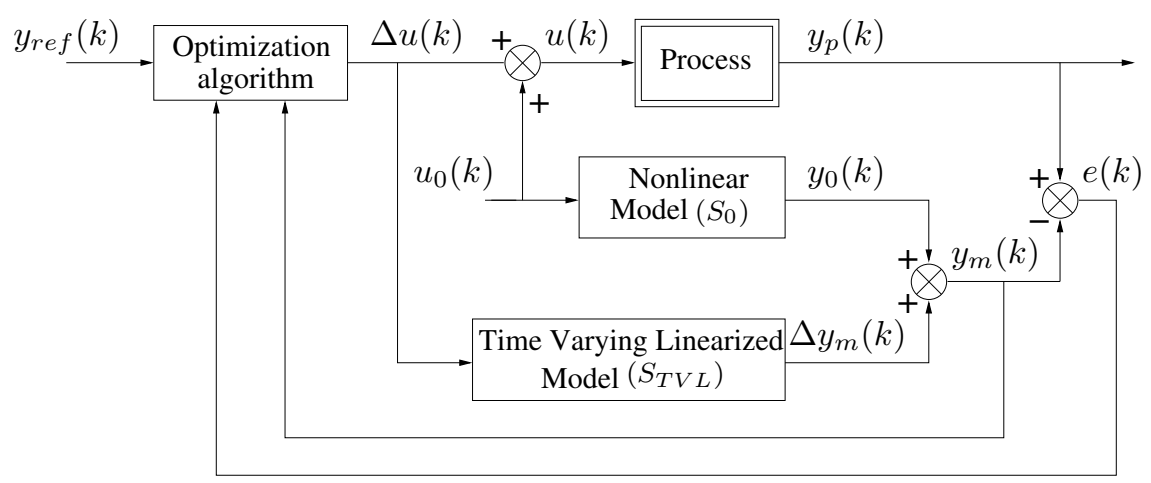

Fig. 3. General Linearized IMC-MPC structure.

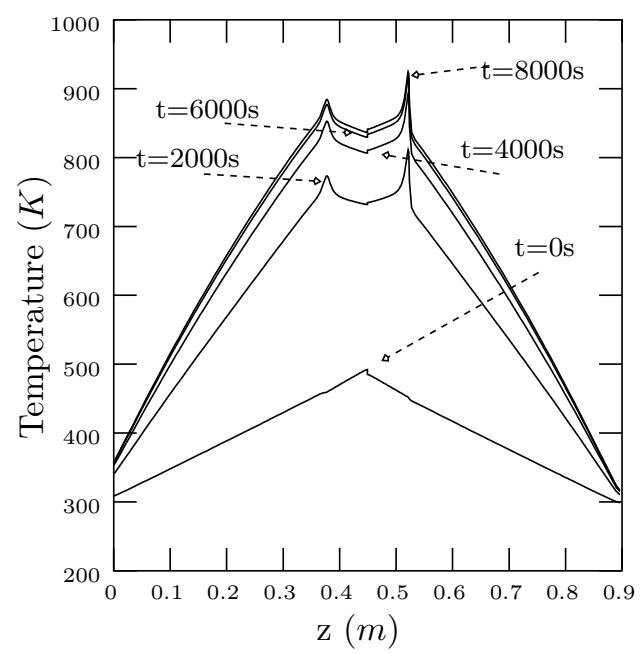

Fig. 4. Open loop temperature profile snapshots in the solid in $\left(S_{N L}\right)$. 


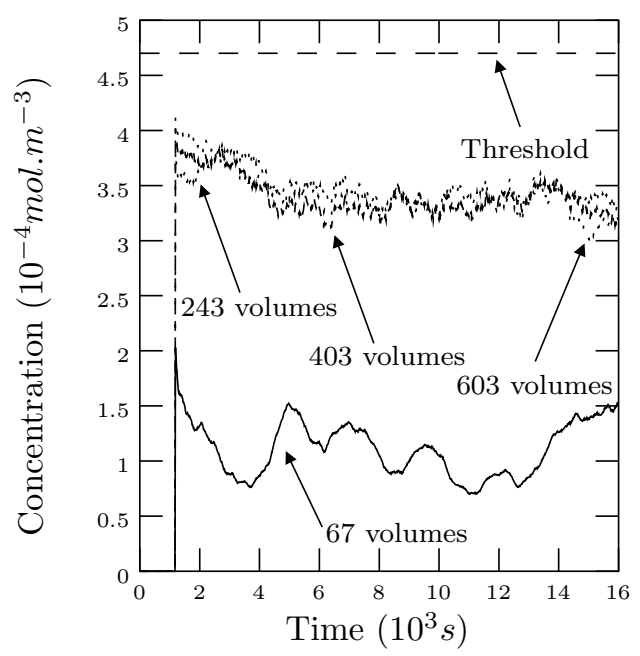

Fig. 5. Run \#1: Mean concentration of pollutant at the process outlet for various finite dimension approximations of $\left(S_{T V L}\right)$.

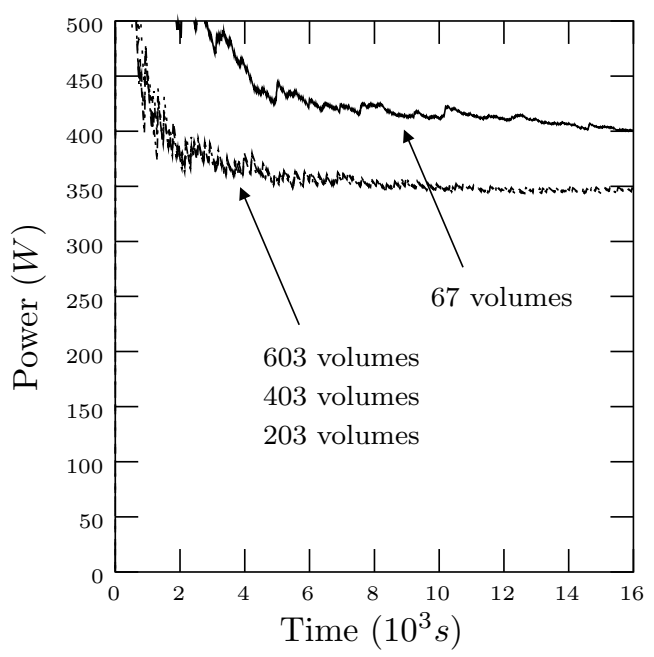

Fig. 6. Run \#1: Mean electrical power for various finite dimension approximations of $\left(S_{T V L}\right)$. 

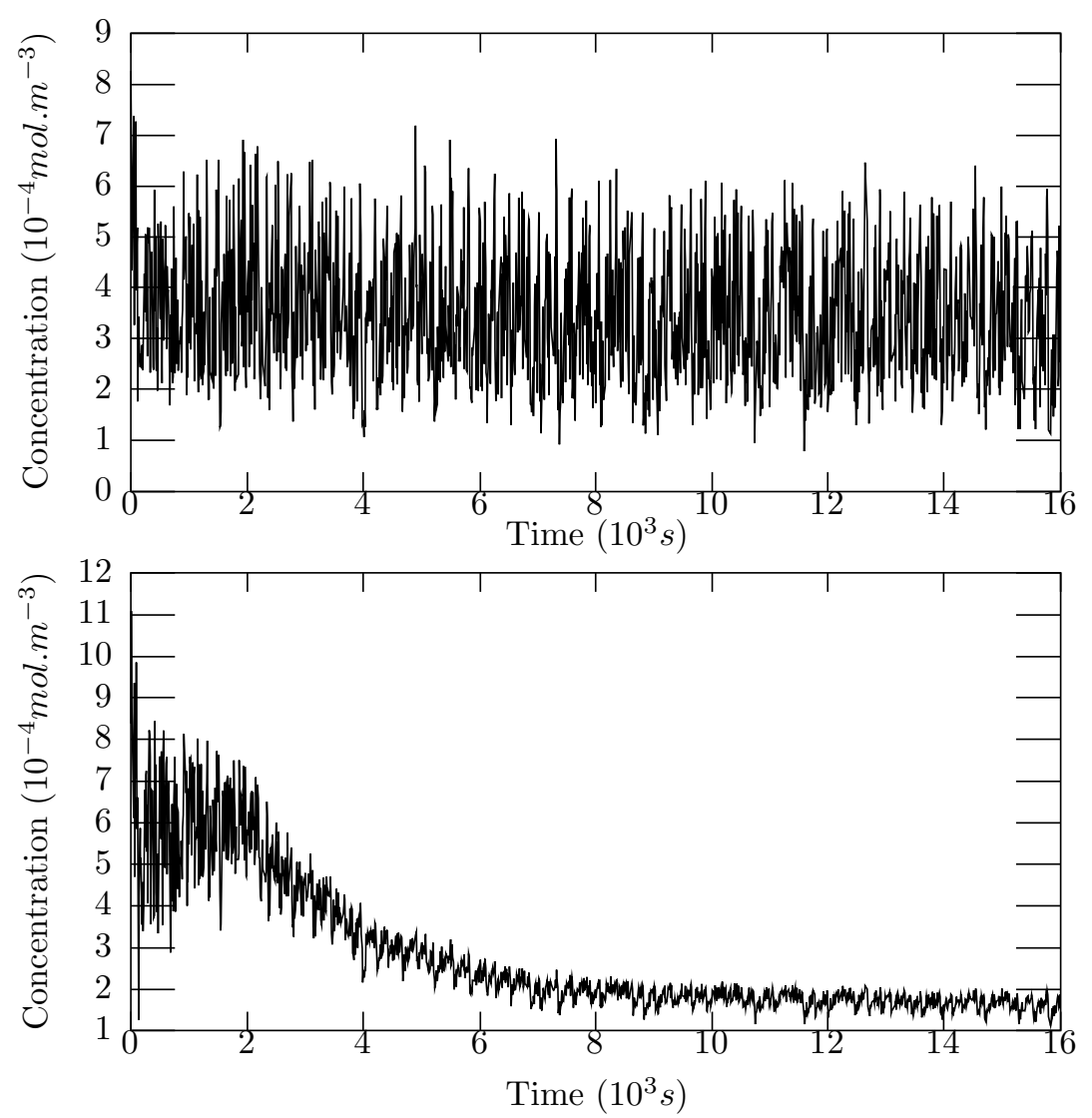

Fig. 7 . Run \#1: Instantaneous concentration of pollutant at the process outlet (top) and at the model outlet (bottom).

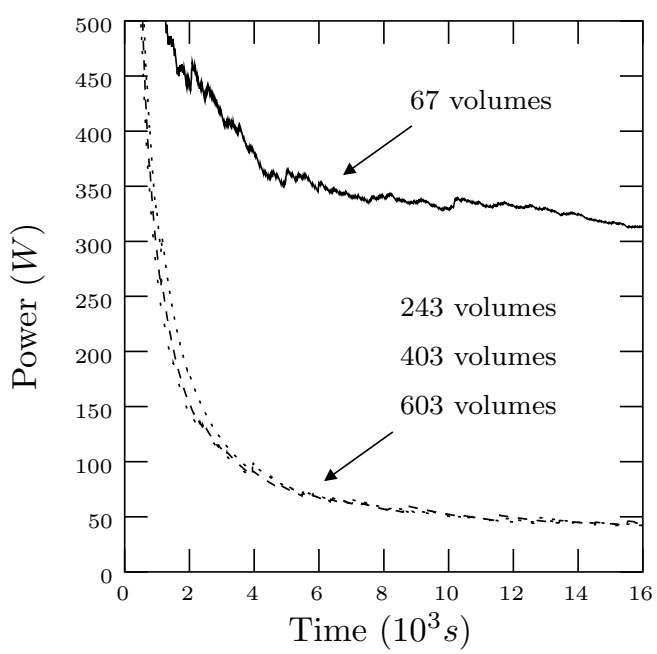

Fig. 8. Run \#2: Mean electrical power for various finite dimension approximations of $\left(S_{T V L}\right)$. 


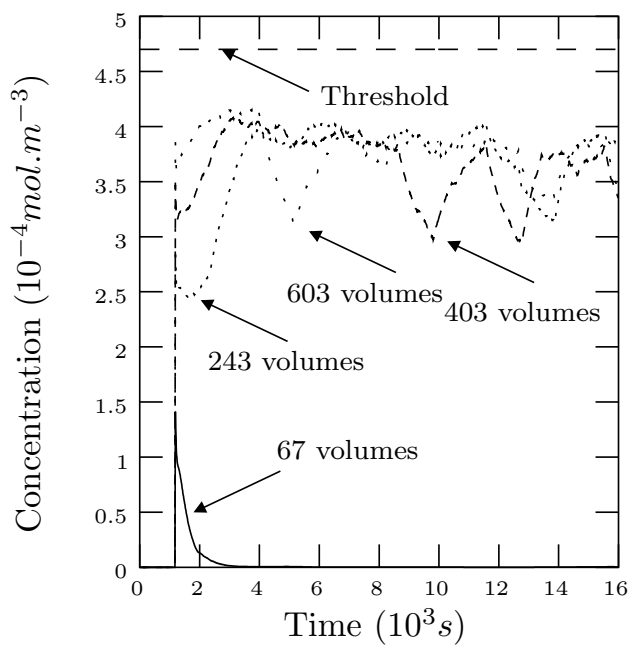

Fig. 9. Run \#2: Mean concentration of pollutant at the process outlet for various finite dimension approximations of $\left(S_{T V L}\right)$.

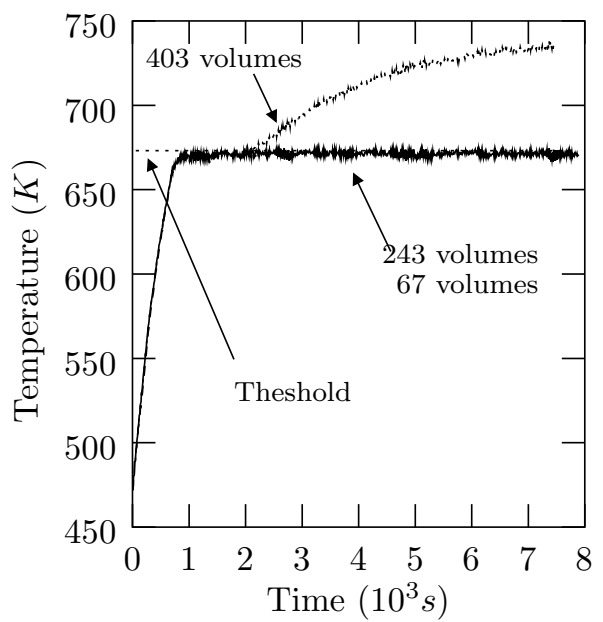

Fig. 10. Run \#3: Maximum temperature inside the reactor for various finite dimension approximations of $\left(S_{T V L}\right)$. 


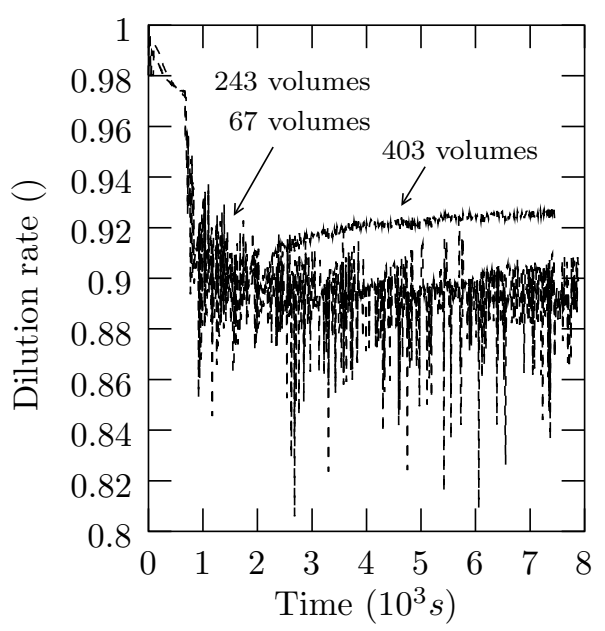

Fig. 11. Run \#3: Dilution rate for various finite dimension approximations of $\left(S_{T V L}\right)$. 


\section{References}

[1] Allgöwer, F., Badgwell, T.A., Qin, J.S., Rawlings, J.B., \& Wright, S.J. (1999). Nonlinear predictive control and moving horizon estimation. In P.M. Frank (Ed.) Advances in control. Highlights of ECC'99, Springer, Berlin.

[2] Armaou, A., \& Christofides, P.D. (2002). Dynamic optimization of dissipative PDE systems using nonlinear order reduction. Chem. Eng. Sci., 57(24), 50835114.

[3] Balas, M.J. (1998). Stable feedback control of linear distributed parameter systems: time and frequency domain conditions. J. Math. Anal. Appl., 225(1), $144-167$.

[4] Budman, H., Kzyonsek, M., \& Silverston, P. (1996). Control of a nonadiabatic packed bed reactor under periodic flow reversal. Canadian J. of Chem. Eng., $74,751-759$.

[5] Christofides, P.D., \& Daoutidis, P. (1997). Finite-dimensional control of parabolic PDE systems using approximate inertial manifolds. J. Math. Anal. Appl., 216(2), 398-420.

[6] Christofides, P.D. (1998). Robust control of hyperbolic PDE systems. Chem. Eng. Sci., 53(1),85-105.

[7] Cittadini, M., Vanni, M., Barresi, A.A., \& Baldi, G. (2001). Simplified procedure for design of catalytic combustors with periodic flow reversal Chem. Eng. Process., 40(3), 255-262. 
[8] Cunill, F., Van de Beld, L., \& Westerterp, K.R. (1997). Catalytic combustion of very lean mixtures in a reverse flow reactor using an internal electrical heater. Ind. Eng. Chem. Res., 36, 4198-4206.

[9] Dufour, P., Couenne, F., \& Touré, Y. (2003). Model predictive control of a catalytic reverse flow reactor, special issue of IEEE Trans. on Control Syst. Technol. on Control of Industrial Spatially Distributed Parameter Processes, 11(5), 705-714.

[10] Eaton, J.W., \& Rawlings, J.B. (1990). Feedback control of chemical processes using on-line optimization techniques. Comput. \& Chem. Eng., 14(4-5), 469-479.

[11] Edouard, D., Hammouri, H., \& Schweich, D. (2004). Observer design for reverse flow reactor. Accepted for publication in AIChE J..

[12] El-Farra, N.H., Armaou, A., \& Christofides, P.D. (2003). Analysis and control of parabolic PDE systems with input constraints. Automatica, 39(4), 715-725.

[13] Fletcher, R. (1987). Practical methods of optimization. John Wiley and Sons.

[14] Friedly, J.C. (1972). Dynamic behavior of processes. Prentice-Hall, Inc.

[15] Godasi, S., Karakas, A., \& Palazoglu, A. (2002). Control of nonlinear distributed parameter processes using symmetry groups and invariance conditions. Comput. \& Chem. Eng., 26(7-8), 1023-1036.

[16] Haynes, T.N, Georgakis, C., \& Caram, H.S. (1995). The design of reverse flow reactors for catalytic combustion systems. Chem. Eng. Sci., 50(3), 401-416.

[17] Hoo, K.A., \& Zheng, D. (2001). Low-order control-relevant models for a class of distributed parameter systems. Chem. Eng. Sci., 56(23), 6683-6710. 
[18] Irizarry-Rivera R., Seider, W.D. (1997). Model-predictive control of the Czochralski crystallization process Part I. Conduction-dominated melt. J. of Crystal Growth, 178(4), 593-611.

[19] Keith, J.M. (2003). Controlling reverse-flow reactors via multiscale transient thermal dispersion. Adv. in Environm. Res., 7(2), 521-535.

[20] Matros,Y.S., \& Bunimovich, G.A. (1996). Reverse-flow operation in fixed bed catalytic reactors. Cat. Rev. Sci. Eng., 38(1), 1-68.

[21] Mayne, D.Q., Rawlings, J.B., Rao, C.V., \& Scokaert, P.O.M. (2000). Constrained model predictive control: stability and optimality. Automatica, 36(6), 789-814.

[22] Morari, M., \& Zafiriou, E. (1989). Robust process control. Dunod.

[23] Morari, M., \& Lee, J.H. (1999). Model predictive control: past, present and future. Comput. \& Chem. Eng., 23(4-5), 667-682.

[24] Nieken, U., Kolios, G., \& Eigenberger, G. (1995). Control of the ignited steady state in authothermal fixed-bed reactors for catalytic combustion. Chem. Eng. Sci., 49(24), 5507-5518.

[25] Nieken, U., Kolios, G., \& Eigenberger, G. (1995). Limiting cases and approximate solutions for fixed-bed reactors with periodic flow reversal. AIChE J., 41(8), 1915-1925.

[26] Palazoglu, A., \& Karakas, A. (2000). Control of nonlinear distributed parameter systems using generalized invariants. Automatica, 36(5), 697-703. 
[27] Patwardhan, A.A., Wright, G.T., \& Edgar, T.F. (1992). Nonlinear modelpredictive control of distributed-parameter systems. Chem. Eng. Sci., 47(4), 721-735.

[28] Polak, E. (1971). Computational Methods in Optimization. Academic Press.

[29] Qin, S.J, \& Badgwell, T.A. (2003). A survey of industrial model predictive control technology. Control Eng. Pract., 11(7), 733-764.

[30] Ramdani, K., Pontier, R., \& Schweich, D. (2001). Reverse low reactor at short switching periods for VOC combustion. Chem. Eng. Sci., 56(4), 1531-1539.

[31] Rawlings, J.B (2000). Tutorial overview of model predictive control. IEEE Control Syst., 20(3), 38-52.

[32] Touré, Y., Biston, J., \& Gilles, G. (1994). Modeling of a distributed parameter process with a variable boundary: application to its control. Chem. Eng. Sci., 49(1), 61-73.

[33] Touré, Y., \& Josserand, L. (1997). An extension of IMC to boundary control of distributed parameter systems. Proc. of the IEEE International Conference on Systems, Man and Cybernetics-CCS, Orlando, USA, 3, 2426-2431.

[34] Van de Beld, B., \& Westerterp, K.R. (1994). Air purification by catalytic oxidation in a reactor with periodic flow reversal. Chem. Eng. Technol., 17, $217-226$.

[35] Zheng, D., \& Hoo, K.A. (2002). Low-order model identification for implementable control solutions of distributed parameter systems. Comput. \& Chem. Eng., 26(7-8), 1049-1076. 
[36] Zheng, D., \& Hoo, K.A. (2004). System identification and model-based control for distributed parameter systems. Comput. $\&$ Chem. Eng., in press. 